\title{
Plasma metabolic characterisation of dairy cows with inactive ovaries and oestrus during the peak of lactation
}

\author{
Yu-Xi Song*, Pan Hu*, Yun-Long Bai, Chang Zhao, Cheng Xia, Chuang Xu \\ College of Animal Science and Veterinary Medicine, \\ Heilongjiang Bayi Agriculture University, Daqing 163319, China \\ xcwlxyf@sohu.com (CX)
}

Received: February 1, 2019

Accepted: July 16, 2019

\begin{abstract}
Introduction: Differential metabolites (DMs) between cows with inactive ovaries (IO) and oestrous (E) cows were screened and metabolic pathways of DMs associated with IO were determined. Material and Methods: Cows at 50 to 60 days (d) postpartum from an intensive dairy farm were randomly selected and allocated into an E group $(n=16)$ or an IO group $(n=16)$ according to a pedometer and rectal examinations. Their plasma samples were analysed by liquid chromatography-mass spectrometry (LC-MS) to compare plasma metabolic changes between the $\mathrm{E}$ and IO groups. Multivariate pattern recognition was used to screen the DMs in the plasma of IO cows. Results: Compared with normal E cows, there were abnormalities in 20 metabolites in IO cows, including a significantly decreased content (VIP $>1, \mathrm{P}<0.05$ ) of cholic acid, $\mathrm{p}$-chlorophenylalanine, and arachidonic acid, and a significantly increased content (VIP $>1, \mathrm{P}<0.05$ ) of tyramine, betaine, L-phenylalanine, L-glutamate, D-proline, L-alanine, and L-pyrophosphate. Five DMs (cholic acid, D-proline, L-glutamate, L-alanine, and L-pyroglutamic acid) with higher variable importance in projection (VIP) values between groups were validated by ELISA with blind samples of re-selected cows (IO, 50 to $60 \mathrm{~d}$ postpartum) and the validated results were consistent with the LC-MS results. Conclusion: The $20 \mathrm{DMs}$ in IO cows during the peak of lactation indicated that the pathogenesis of IO was involved in complex metabolic networks and signal transduction pathways. This study provides a basis for further exploration of the pathogenesis and prevention of IO in cows in the future.
\end{abstract}

Keywords: dairy cows, metabolites, inactive ovary, liquid chromatography-mass spectrometry.

\section{Introduction}

Over the past few decades, the reproductive performance of dairy cows has experienced a downward trend worldwide (30). In China, reproductive disorders have become an important factor that restricts the development of the dairy industry. In addition, the number of cows eliminated due to reproductive disorders has continued to rise yearly. Anoestrus, which accounts for about $26.68 \%$ of reproductive disorders, is one of the most important reasons for the fall in dairy cattle reproductivity (28).

Inactive ovaries (IO), one of the most common types of postpartum anoestrus in cows, is caused by follicular growth disorders and transient functional disturbances of the ovaries (20). Deficient or nonexistent follicle cyclicity as an anoestrus cause is also IO (20). Many factors can cause or aggravate IO in cows, including low nutrient intake, high milk yield (MY), inadequate body condition score (BCS), and poor management of stress and diseases (2). However, nutritional status has a far-reaching impact on the reproductive performance of cows, as there is a close relationship between postpartum ovarian activity and energy balance. Negative energy balance (NEB) is an altered metabolic state in high-yielding cows that occurs during the first few weeks postpartum when energy demands for lactation and maintenance exceed the energy supply from dietary intake. Butler et al. (6) found that NEB inhibited the pulsed secretion of gonadotropin releasing hormone $(\mathrm{GnRH})$ from the hypothalamus and luteinising hormone (LH) from the pituitary gland which reduced the responsiveness of an ovary to LH. In the absence of LH, the growth of the ovary and establishment of dominant follicles are inhibited, which hinders the normal growth and 
development of dominant follicles, eventually leading to the disappearance of ovarian periodic activity and the occurrence of IO.

The lack of energy during early lactation seriously affects the size of the dominant follicles and is a determinant of whether the final ovulation will be successful, although the underlying mechanisms remain unknown. McLaughlin and McIver (17) and Fortune (9) found that malnutrition was a primary cause of $\mathrm{IO}$, because of the lack of certain growth peptide factors or cell activins which had a positive effect on follicular growth. Moreover, Salesh et al. (21) reported that insulin-like growth factor-1 (IGF-1), thyroxine, and energy-related metabolites were positively correlated with IO and closely related to reproduction. However, these studies focused on the effect of a specific endocrine or energy pathway-related factor on the normal ovarian cycle and there is only a report on metabolic profiles of postpartum dairy cows with ovarian inactivity using ${ }^{1} \mathrm{H}$-nuclear magnetic resonance spectroscopy. Prior to this article, the full extent of the metabolic changes in dairy cows with IO which are induced by NEB was still unclear.

Therefore, in this study, liquid chromatographymass spectrometry (LC-MS) was used to identify the differential metabolites (DMs) in the plasma between IO and E cows, and ELISA was used to validate the five identified DMs. The aim of this study was to clarify the metabolic profile caused by NEB in dairy IO cows and to provide a new direction for the further study of the aetiology of IO postpartum.

\section{Material and Methods}

Animals. All cows from an intensive dairy farm in the Heilongjiang Province of China were maintained in free-stall housing with continuous access to fresh water and were milked three times per day (d). Total mixed rations (TMR) during early lactation were formulated in accordance with National Research Council (2001) standards. The TMR consisted of concentrated feed, silage, hay, and fat. Feed analysis showed $55.6 \%$ of dry matter, $16 \%$ of crude protein, $5.6 \%$ of fat, $20.58 \mathrm{MJ} / \mathrm{kg}$ of net energy for lactation, $0.56 \%$ of $\mathrm{Ca}$, and $0.36 \%$ of $P$.

A total of 32 Holstein cows at 50 to $60 \mathrm{~d}$ postpartum without oestrus synchronisation, uterine disorders, ovarian abnormalities, or other diseases were randomly chosen and assigned appropriately to the oestrous group $(E, n=16)$ or the inactive ovary group (IO, $n=16)$. The latter contained cows defined by an Afitag pedometer (Afimilk 0418A09QPDX, Israel) for detection of oestrous behaviour and by rectal examination by experienced personnel for follicle development. All cows were 2-4 years old, with parity of 1-3.

Sample collection. At 50 to $60 \mathrm{~d}$ postpartum, before milking and fasting in the morning, $10 \mathrm{~mL}$ of blood was collected from the jugular vein into an anticoagulant tube and mixed evenly with three drops of heparin sodium. Anticoagulated blood was centrifuged at $1,500 \times \mathrm{g}$ for $5 \mathrm{~min}$ and the supernatant was placed into a $1.5 \mathrm{~mL}$ Eppendorf tube. Then, the supernatant was centrifuged at $12,000 \times \mathrm{g}$ for $5 \mathrm{~min}$ and $500 \mu \mathrm{L}$ of plasma was placed into a $1.5 \mathrm{~mL}$ Eppendorf tube and stored at $-80^{\circ} \mathrm{C}$ for biochemical analysis of glucose (Glu), nonesterified fatty acid (NEFA), and $\beta$-hydroxybutyric acid (BHBA), or for LC-MS analysis. Levels of plasma Glu, NEFA, and BHBA were measured directly using a Synchron DXC800 fully automatic biochemistry analyser (Beckman Coulter, USA).

LC-MS Sample preparation. Before LC-MS detection, 32 plasma samples from $\mathrm{E}$ and $\mathrm{IO}$ cows were thawed at room temperature. L-2-chlorophenylalanine $(10 \mu \mathrm{L})$ was used as an internal standard. Briefly, $100 \mu \mathrm{L}$ of each sample was mixed with $400 \mu \mathrm{L}$ of methanol:acetonitrile $(1: 1, \mathrm{v} / \mathrm{v})$ and vortexed for $30 \mathrm{~s}$, sonicated for $10 \mathrm{~min}$ in an ice bath, and centrifuged at $12,000 \times \mathrm{g}$ for $15 \mathrm{~min}$ at $4^{\circ} \mathrm{C}$. After standing for $1 \mathrm{~h}$ at $20^{\circ} \mathrm{C}, 400 \mu \mathrm{L}$ of the supernatant was rotated and evaporated in a vacuum concentrator at room temperature to dryness. The dry extract was then redissolved in $100 \mu \mathrm{L}$ of acetonitrile: $\mathrm{H}_{2} \mathrm{O}(1: 1, \mathrm{v} / \mathrm{v})$, vortexed for $30 \mathrm{~s}$, sonicated for $10 \mathrm{~min}$ in an ice bath, and centrifuged at $12,000 \times \mathrm{g}$ for $15 \mathrm{~min}$ at $4^{\circ} \mathrm{C}$ to remove insoluble debris. From each treated sample, $5 \mu \mathrm{L}$ of the supernatant was mixed as a quality control (QC) sample and $70 \mu \mathrm{L}$ was aliquoted for LC-MS detection (12).

Detection analysis. Detection was performed using an Agilent 1290 Infinity II LC system (Agilent Technologies, USA) coupled to a TripleTOF 6600 System (Q-TOF; AB Sciex, USA).

The following ultra-high performance LC conditions were used in an Acquity BEH Amide UPLC column $(1.7 \mu \mathrm{m}, 2.1 \times 100 \mathrm{~mm}$; Waters, USA): mobile phase A, composed of $25 \mathrm{mM}$ ammonium acetate and $25 \mathrm{mM}$ ammonia aqueous solution; mobile phase $\mathrm{B}$, and acetonitrile. The gradient elution programme was as follows: $0 \mathrm{~min}, 15 \% \mathrm{~A} / 85 \% \mathrm{~B} ; 1 \mathrm{~min}, 15 \% \mathrm{~A} / 85 \% \mathrm{~B}$; $12 \mathrm{~min}, 35 \% \mathrm{~A} / 65 \% \mathrm{~B} ; 12.1 \mathrm{~min}, 60 \% \mathrm{~A} / 40 \% \mathrm{~B}$; $15 \min , 60 \% \mathrm{~A} / 40 \% \mathrm{~B} ; 15.1 \mathrm{~min}, 15 \% \mathrm{~A} / 85 \% \mathrm{~B}$; and $20 \mathrm{~min}, 15 \% \mathrm{~A} / 85 \% \mathrm{~B}$. The flow rate was $0.3 \mathrm{~mL} / \mathrm{min}$ and the sample injection volume was $2 \mu \mathrm{L}$.

The mass spectrometer was used for its ability to acquire level one and two MS spectra on an informationdependent basis using control software (Analyst TF 1.7; AB Sciex) during LC-MS analysis. In each data acquisition cycle, the strongest 100 or more molecular ions were selected to collect the corresponding secondary mass spectrometry data. The collision energy was $35 \mathrm{~V}$ and 15 secondary MS spectrograms were collected at an accumulation time of $50 \mathrm{~ms}$ each (4). The electrospray ionisation (ESI) source conditions were set as follows: ion source gas $1-60 \mathrm{~Pa}$; ion source gas $2-60 \mathrm{~Pa}$; curtain gas - $30 \mathrm{~Pa}$; source temperature $-550^{\circ} \mathrm{C}$; and ion spray voltage floating $-5,500 \mathrm{~V}$ (positive mode) and $-4,500 \mathrm{~V}$ (negative mode). 
Data processing. The raw LC-MS data were converted to mzXML format using ProteoWizard MS Convert version 3.0.4146 (http://proteowizard. sourceforge.net). At the same time, a chromatogram of total ion current (TIC) was produced using PeakView version 1.1 (AB Sciex). The mzXML files were processed using XCMS online software (https://xcmsonline.scripps.edu/landing_page.php?pgco ntent $=$ mainPage) for peak detection, retention time correction, peak alignment, and other data processing to filter out noisy data (23). The method was as follows: compounds in the QC samples with a relative standard deviation $>30 \%$ were deleted, peak area data in a single group or all groups with a null value $\leq 50 \%$ were retained, all missing values in the raw data were padded with the least one-half method, and the filtered data was standardised using the area normalisation method.

Identification and matching of compounds. Data for compound identification were processed and matched using the Xcms4dda and xcms4lipid programmes, and self-built libraries based on XCMS development with parameters of minfrac $=0.5$ and cutoff $=0.8$. The secondary data were screened and the peaks that satisfied the principle of forward and reverse were retained as long as one was identified. Then, the peaks of the level one and two data were matched according to $\mathrm{mz}$ tolerance $\pm 25 \mathrm{ppm}$ (23).

Multivariate statistical analysis. The analysis included principal component analysis (PCA) and orthogonal partial least-squares discriminant analysis (OPLS-DA), which were performed for normalised data using SIMCA-P version 14.0 (Umetrics AB, Sweden). PCA was used to assess the overall metabolic differences among groups and the variability between samples within a group. OPLS-DA was also based on a unit variance scaling strategy to maximise the highlights of the internal model within the differences between groups (27).

DM screening and pathway analysis. DMs between groups were screened by OPLS-DA (variable importance in projection (VIP) $>1$ ) combined with the $t$-test $(\mathrm{P}<0.05)$. The Kyoto Encyclopaedia of Genes and Genomes database (http://www.kegg.jp) was accessed to identify relevant pathways of the DMs and the MetaboAnalyst 3.0 web server (http://www. metaboanalyst.ca) was used to analyse the metabolic pathways.

ELISA. At 50 to $60 \mathrm{~d}$ postpartum, cows were re-selected to validate the five DMs with the highest VIP values between the $\mathrm{E}$ and $\mathrm{IO}$ groups. The blind samples of re-selected cows were from 16 oestrous cows and 16 inactive ovary cows and their collection and preparation were consistent with the procedure followed for the LC-MS analysis. The quantities of cholic acid, D-proline, L-alanine, L-glutamate, and L-pyroglutamic acid were detected using ELISA kits (Shanghai Best Biotechnology Co., China) in accordance with the manufacturer's instructions.
Statistical analysis. The independent samples $t$-test was used to compare clinical data (age, parity, BCS, MY, BHBA, NEFA, and Glu) and ELISA data (five DMs) of cows between the E and IO groups using IBM SPSS Statistics for Windows version 19.0 (IBM Corp., USA). $\mathrm{P}<0.05$ and $\mathrm{P}<0.01$ were considered significant and very significant, respectively.

\section{Results}

LC-MS clinical data. The age, parity, BCS, and plasma concentrations of Glu, NEFA, and BHBA are listed in Table 1. There were a significant decrease in BCS and a significant increase in plasma NEFA of the IO group compared with those of the $\mathrm{E}$ group. It suggested that cows with inactive ovaries might experience greater fat mobilisation induced by a negative energy balance during early lactation.

TIC chromatograms of QC samples. The TIC chromatograms of QC samples of the E and IO groups in two modes (ESI+/ESI-) are shown in Fig. 1. The chromatograms acquired by LC-MS were overlaid using PeakView version 1.1. The results showed that retention times and peak intensities of the QC samples had good reproducibility in the chromatograms in both modes (ESI+ and ESI-), which indicated that the test process was stable and reliable, including the pre-sample processing and instrument analysis systems.

Multivariate statistical analysis. PCA was performed first to identify intrinsic patterns in the data set and to gain an overview of variation between the $\mathrm{E}$ and IO groups. The results showed that $\mathrm{R}^{2} \mathrm{X}=0.493$ and $\mathrm{Q}^{2}=0.21$, indicating that PCA lacked the power to sufficiently separate the two groups, as shown in Fig. 2. OPLS-DA was then performed to gain better group separation and to reveal DMs between the two groups. In the OPLS-DA score plot of Fig. 3a, the two groups are well separated $\left(\mathrm{R}^{2} \mathrm{Y}=0.942, \mathrm{Q}^{2}=0.662\right)$. The quality of the OPLS-DA model was checked by sevenfold cross-validation. The results of OPLS-DA combined with those of the permutation tests in Fig. $3 \mathrm{~b}$ suggest that the established model is effective, indicating that there were significant differences in metabolites between the groups.

DMs and pathway analysis. As shown in Table 2, 9 DMs were screened in the ESI+ model and 15 in the ESI- model. After the duplicate metabolites of the two models were removed, there were $20 \mathrm{DMs}$ in plasma from IO cows. The concentrations of plasma cholic acid, p-chlorophenylalanine, and arachidic acid were significantly decreased (VIP $>1, \mathrm{P}<0.05$ ), while those of plasma tyramine, betaine, L-phenylalanine, L-glutamate, N6,N6,N6-trimethyl-L-lysine, D-proline, L-pyro-glutamic acid, L-citrulline, $\gamma$-L-glutamyl-L-valine, L-leucine, L-valine, L-isoleucine, L-alanine, cytidine, creatine, phenylacetylglycine, and $\alpha-\mathrm{N}$-phenylacetyl-L-glutamine were significantly increased (VIP $>1, \mathrm{P}<0.05$ ). These 
metabolites were involved mainly in glycometabolism, lipid metabolism, and protein synthesis. More specifically, the metabolic pathways included phenylalanine, tyrosine, and tryptophan biosynthesis and the metabolisms of glutathione (GSH), arginine and proline, D-glutamine and D-glutamate, and alanine, aspartate acid, and glutamate (Fig. 4).

ELISA. Clinical data. As shown in Table 1, the age, parity, BCS, and energy balance status in samples of blind-selected cows were similar to those of cows used for LC-MS analyses.

DMs. The 20 DMs were further screened to identify those closely related to the occurrence of ovary inactivity. As shown in Table 3, there were five validated metabolites with higher VIP values. As compared to group E cows, D-proline, L-alanine, and L-pyroglutamic acid levels were significantly higher in group IO cows, while there were no significant differences in L-glutamate or cholic acid levels between the two groups. However, as compared to group E cows, those in the IO group had lower levels of cholic acid and higher levels of L-glutamate. These findings suggest that the quantitative validation results by ELISA were consistent with those obtained by LC-MS analysis.

Table 1. Clinical data in two groups of cow samples at 50 to $60 \mathrm{~d}$ postpartum for LC-MS detection and for ELISA validation

\begin{tabular}{lllll}
\hline \multirow{2}{*}{ Parameters } & \multicolumn{2}{l}{ Cow samples for LC-MS detection } & \multicolumn{2}{l}{ Cow samples for ELISA validation } \\
\cline { 2 - 5 } & E & IO & E & IO \\
\hline No. & 16 & 16 & $3.29 \pm 0.34$ & $3.23 \pm 0.55$ \\
Age & $3.58 \pm 1.40$ & $3.07 \pm 1.26$ & $1.91 \pm 0.28$ & $1.86 \pm 0.55$ \\
Parity & $2.27 \pm 1.16$ & $1.88 \pm 1.09$ & $2.84 \pm 0.23$ & $2.66 \pm 0.21^{*}$ \\
BCS & $2.88 \pm 0.29$ & $2.69 \pm 0.23^{*}$ & $33.37 \pm 4.48$ & $34.90 \pm 4.64$ \\
MY $(\mathrm{Kg} / \mathrm{d})$ & $33.56 \pm 4.48$ & $34.68 \pm 5.00$ & $3.53 \pm 0.51$ & $3.39 \pm 0.39$ \\
Glu $(\mathrm{mmol} / \mathrm{L})$ & $3.40 \pm 0.34$ & $3.36 \pm 0.38$ & $0.21 \pm 0.08$ & $0.30 \pm 0.13^{*}$ \\
NEFA $(\mathrm{mmol} / \mathrm{L})$ & $0.21 \pm 0.05$ & $0.32 \pm 0.12^{* *}$ & $0.54 \pm 0.17$ & $0.60 \pm 0.28$ \\
BHBA $(\mathrm{mmol} / \mathrm{L})$ & $0.53 \pm 0.21$ & $0.63 \pm 0.18$ & &
\end{tabular}

$* \mathrm{P}<0.05 ; * * \mathrm{P}<0.01 ; \mathrm{E}$ - oestrous cows; $\mathrm{IO}$ - inactive ovary cows; $\mathrm{BCS}$ - body condition score; $\mathrm{MY}$ milk yield per day; Glu - glucose; NEFA - non-esterified fatty acid; BHBA - $\beta$-hydroxybutyric acid; samples for LC-MS detection were from 16 oestrous and 16 inactive ovary cows; cow samples for ELISA validation were from 16 oestrous and 16 inactive ovary cows re-selected blindly and these samples were taken and prepared in the same way as those used for LC-MS detection

Table 2. Differential plasma metabolites of IO cows and normal E cows screened by LC-MS

\begin{tabular}{|c|c|c|c|c|c|c|c|}
\hline No. & Metabolites & mzmed & rtmed & VIP value & P value & $\mathrm{FC}$ & Mode \\
\hline 1 & Tyramine & 120.0801 & 203.3150 & 1.0774 & 0.0078 & 1.3528 & ESI+ \\
\hline 2 & Betaine & 118.0855 & 288.9015 & 1.3930 & 0.0013 & 1.4872 & ESI+ \\
\hline 3 & L-phenylalanine & 166.0855 & 203.3220 & 1.0598 & 0.0099 & 1.3254 & ESI+ \\
\hline 4 & L-glutamate & 148.0594 & 508.3525 & 1.7459 & 0.0059 & 1.5415 & ESI+ \\
\hline 5 & N6,N6,N6-trimethyl-L-lysine & 189.1587 & 802.7070 & 1.5786 & 0.0003 & 1.4007 & ESI+ \\
\hline 6 & D-proline & 116.0703 & 315.4230 & 1.4542 & 0.0007 & 1.2543 & ESI+ \\
\hline 7 & L-pyroglutamic acid & 130.0490 & 508.3120 & 1.4025 & 0.0050 & 1.2130 & ESI+ \\
\hline 8 & Cholic acid & 391.2832 & 46.9290 & 1.8280 & 0.0003 & 0.6327 & ESI+ \\
\hline 9 & p-chlorophenylalanine & 200.0463 & 243.4400 & 1.2859 & 0.0024 & 0.7587 & ESI+ \\
\hline 10 & L-phenylalanine & 164.0719 & 203.9900 & 1.2468 & 0.0144 & 1.4683 & ESI- \\
\hline 11 & L-citrulline & 349.1815 & 485.9170 & 1.1153 & 0.0177 & 1.6022 & ESI- \\
\hline 12 & L-glutamate & 146.0457 & 510.6010 & 1.8904 & 0.0085 & 1.4992 & ESI- \\
\hline 13 & ү-L-glutamyl-L-valine & 245.1133 & 468.3435 & 1.6628 & 0.0038 & 1.5038 & ESI- \\
\hline 14 & Arachidic acid & 311.2936 & 50.1280 & 1.5495 & 0.0431 & 0.6241 & ESI- \\
\hline 15 & L-leucine & 130.0878 & 219.6660 & 1.4373 & 0.0073 & 1.4759 & ESI- \\
\hline 16 & L-valine & 116.0717 & 290.1260 & 1.5806 & 0.0032 & 1.5261 & ESI- \\
\hline 17 & L-pyroglutamic acid & 128.0356 & 281.0020 & 1.9003 & 0.0025 & 1.6062 & ESI- \\
\hline 18 & L-isoleucine & 130.0877 & 240.2465 & 1.7346 & 0.0010 & 1.6988 & ESI- \\
\hline 19 & L-alanine & 88.0408 & 388.2320 & 1.9019 & 0.0000 & 1.3377 & ESI- \\
\hline 20 & D-proline & 114.0560 & 315.8370 & 1.9989 & 0.0003 & 1.3718 & ESI- \\
\hline 21 & Cytidine & 302.0975 & 208.5070 & 1.1421 & 0.0218 & 1.3280 & ESI- \\
\hline 22 & Creatine & 261.1303 & 388.7810 & 1.5459 & 0.0008 & 1.6097 & ESI- \\
\hline 23 & Phenylacetylglycine & 192.0663 & 103.3500 & 1.7514 & 0.0001 & 1.7482 & ESI- \\
\hline 24 & $\alpha$-N-phenylacetyl-L-glutamine & 385.1378 & 103.4960 & 1.6689 & 0.0003 & 1.8358 & ESI- \\
\hline
\end{tabular}

FC - fold change: The ratio of the average of the IO group (inactive ovary cows, $\mathrm{n}=16$ ) to the E group (oestrous cows, $\mathrm{n}=16$ ), showing the up $(>1)$ and down $(<1)$ relationship of metabolites between inactive ovary cows and oestrous cows; VIP - variable importance in the projection 
Table 3. The ELISA validation of 5 differential metabolites between normal oestrous cows and inactive ovary cows

\begin{tabular}{lll}
\hline Parameters & E & IO \\
\hline No. & 16 & 16 \\
Cholic acid, pmol/L & $59.85 \pm 11.56$ & $52.75 \pm 11.88$ \\
D-proline, $\mathrm{ng} / \mathrm{mL}$ & $37.84 \pm 5.06$ & $41.53 \pm 5.14^{*}$ \\
L-alanine, $\mathrm{ng} / \mathrm{mL}$ & $91.44 \pm 7.24$ & $97.52 \pm 7.12^{*}$ \\
L-glutamate, $\mathrm{ng} / \mathrm{mL}$ & $22.50 \pm 3.60$ & $24.07 \pm 2.72$ \\
L-pyroglutamic acid, ng/mL & $3.23 \pm 0.52$ & $3.60 \pm 0.48^{*}$ \\
\hline
\end{tabular}

$* \mathrm{P}<0.05 ; \mathrm{E}$ - oestrous cows; IO - inactive ovary cows
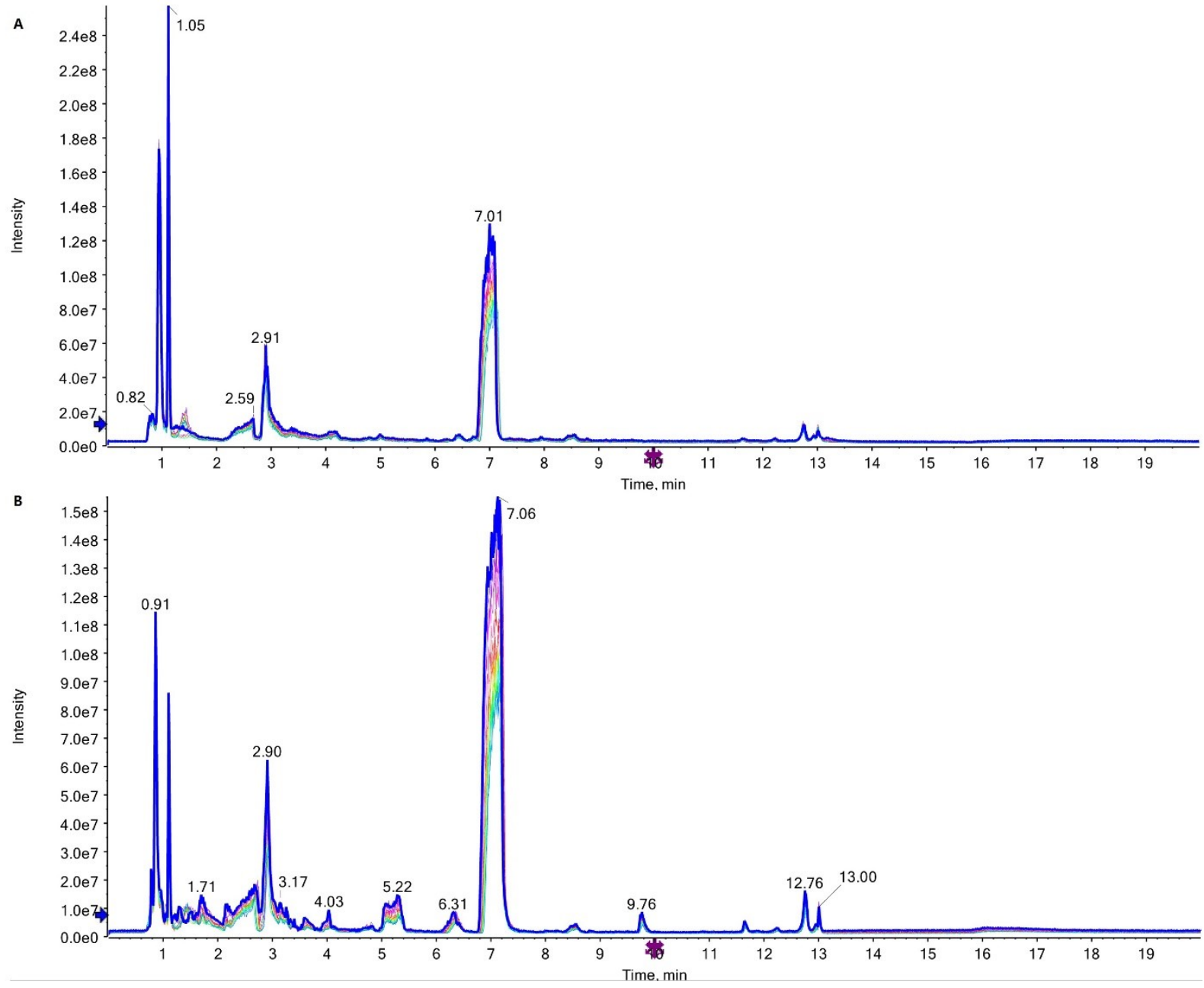

Fig. 1. The TIC (total ion count) of the QC (quality control) samples from the IO (inactive ovary cow) and E (oestrous cow) groups by LC-MS; $\mathrm{A}-\mathrm{ESI}+$ (positive ion of electrospray ionisation) mode; B - ESI- (negative ion of electrospray ionisation) mode

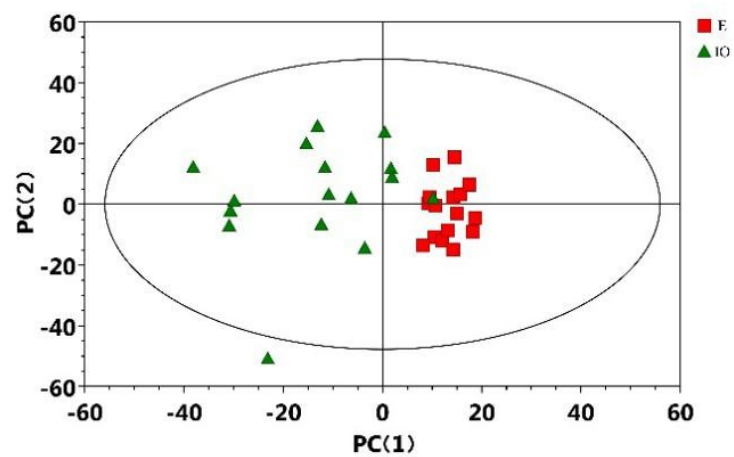

Fig. 2. PCA score plot based on LC-MS spectra of plasma of dairy cows obtained from the $\mathrm{E}$ (oestrous cow) and IO (inactive ovary cow) groups 

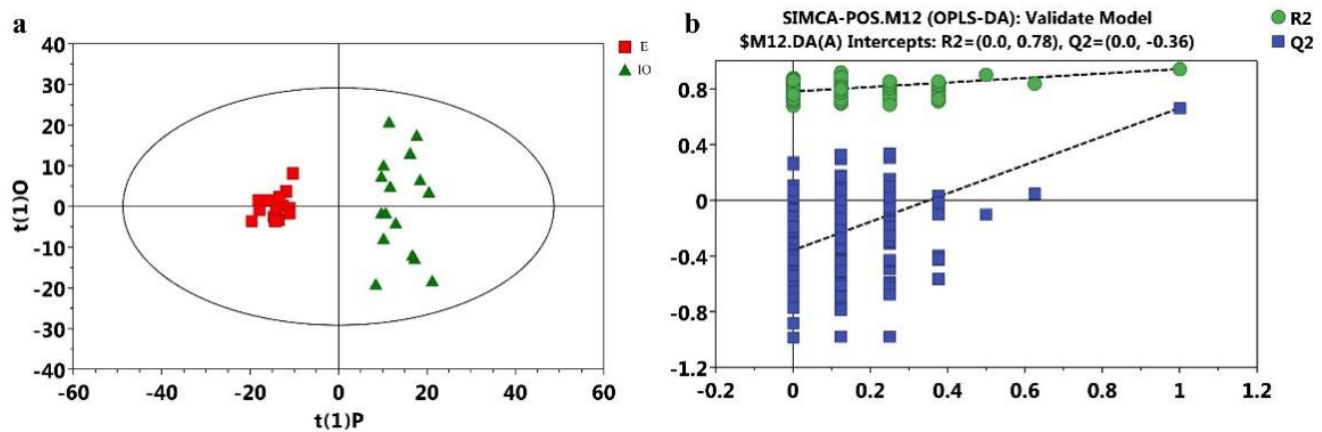

Fig. 3. The OPLS-DA score plot (a) and permutations test plot (b) based on LC-MS spectra of plasma of dairy cows obtained from the E (oestrous cow) and IO (inactive ovary cow) groups
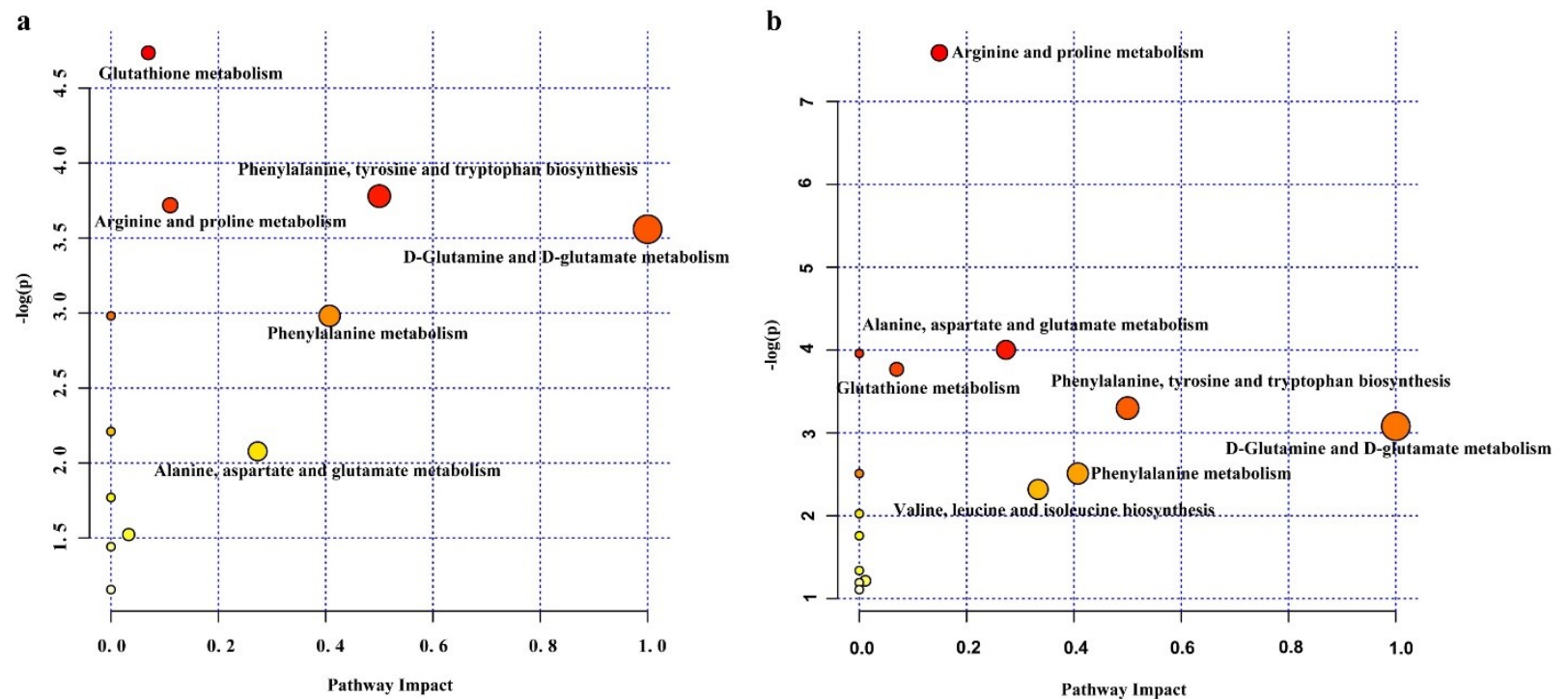

Fig. 4. Pathway analysis of differential plasma metabolites in a - ESI+ (positive ion of electrospray ionisation); and $b-\mathrm{ESI}-($ negative ion of electrospray ionisation) modes between the E (oestrous cow) and IO (inactive ovary cow) groups in LC-MS analysis. The sizes of the bubbles are proportional to the impact of each pathway, with colour denoting the significance from the highest in red to the lowest in white

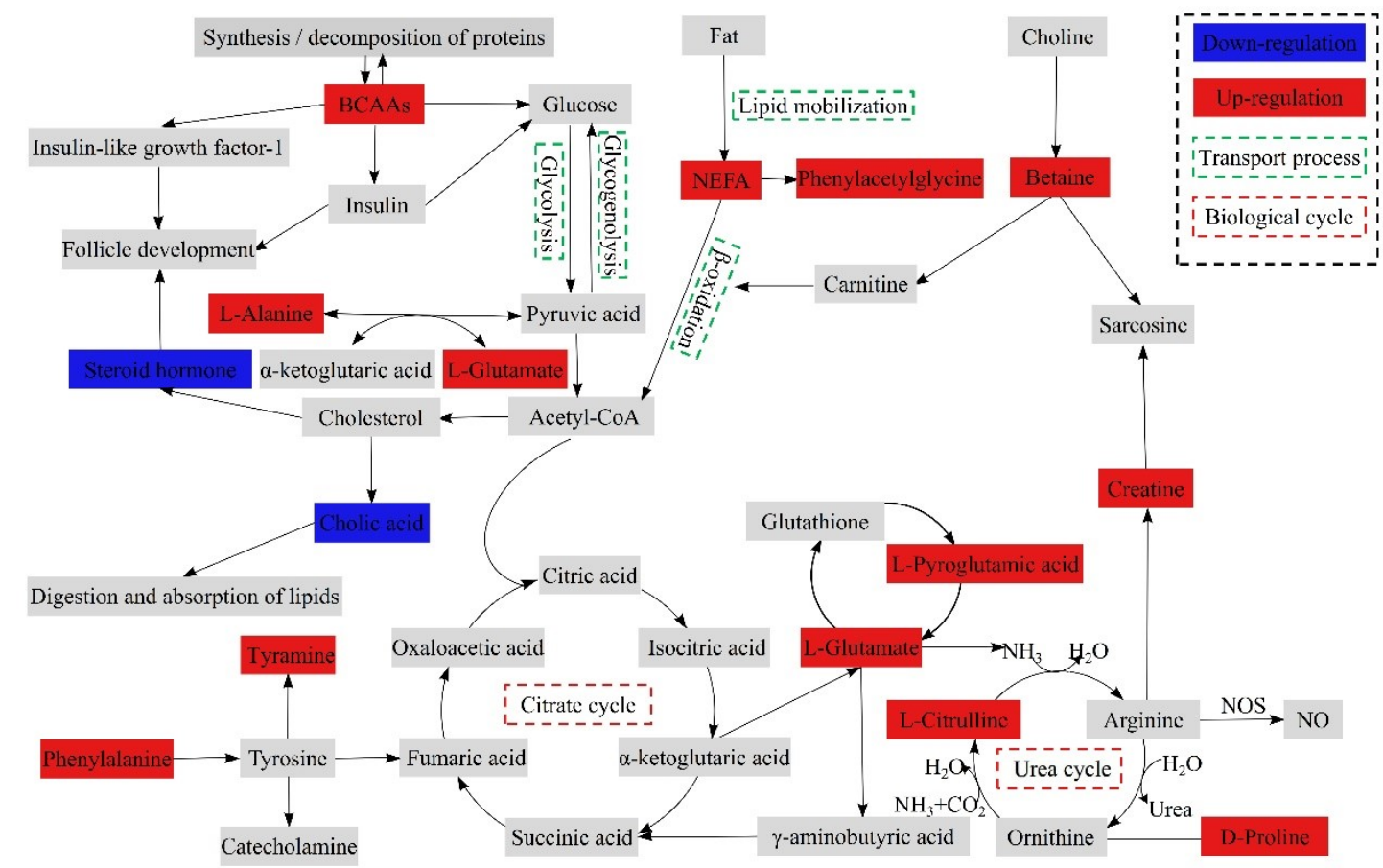

Fig. 5. Summarised pathways of differential metabolites from the IO (inactive ovary cow) and E (oestrous cow) groups. Down-regulation and upregulation refer to the IO group compared to the E group. The pathways of differential metabolites were from the Kyoto Encyclopedia of Genes and Genomes database (http://www.kegg.jp) and the MetaboAnalyst 3.0 web server (http://www.metaboanalyst.ca) 


\section{Discussion}

In recent years, metabolomic technology has been widely applied in many fields and has become an important method in the study of disease pathogenesis and potential biomarkers. In this study, LC-MS technology was used to analyse the plasma metabolite profile of cows with IO resulting from postpartum NEB. The importance of the screened DMs and their metabolic pathways are summarised in Fig. 5. The higher VIP subset DMs were further validated to explore potential relationships with NEB and IO.

Effect of lipid metabolism on IO. Betaine is a quaternary ammonium salt alkaloid that is an important methyl donor and promotes fat metabolism, while inhibiting fat deposition. Betaine can provide the methyl necessary for the synthesis of carnitine, which is an important material in fatty acid $\beta$-oxidation. Carnitine transports fatty acyl-CoA formed by fatty acid activation into the mitochondrial matrix through the mitochondrial inner membrane to conduct $\beta$-oxidation. Therefore, fatty acid $\beta$-oxidation is mainly governed by fatty acyl-CoA entering the mitochondria, and carnitine acyltransferase I is in turn the major enzyme that controls fatty acyl-CoA mitochondrial access. When the body exists in a disease state or experiences glycometabolism disorders, the use of Glu is limited, but with the enhancement of fatty acid oxidation for energy, the activity of carnitine acyltransferase I also increases (5). In this study, at 50 to $60 \mathrm{~d}$ postpartum, plasma NEFA levels in IO cows were significantly higher than those in oestrous cows, indicating that fat mobilisation of IO cows was still stronger than that of oestrous cows. However, plasma betaine levels were increased in the IO cows, because more dynamic fat mobilisation as induced by NEB required high levels of carnitine to transport the fatty acids into the mitochondrial matrix for $\beta$-oxidation to provide energy.

Arachidic acid is a long-chain fatty acid that is absorbed in the digestive tract. Studies have shown that the addition of dietary fat to feed had a certain positive effect on the reproductive performance of dairy cows. A meta-analysis conducted by Staples et al. (25) found that $11(55 \%)$ of 20 studies reported that the first insemination conception rate and total conception rate were significantly improved (by an average of $17 \%$ ) by the addition of dietary fatty acids. The arachidic acid content fell in the IO cows, suggesting that fat intake was smaller or digestion and absorption were less effective than in the E cows, or that utilisation and consumption of fat was increased in the IO cows: either scenario would result in lower reproductive performance. Cholic acid is a free bile acid that is synthesised from cholesterol in the liver, and cholesterol is the precursor of steroid hormones synthesised in the adrenal cortex, testes, and ovaries. The addition of dietary cholesterol to guinea pigs increased the synthesis of bile acids (11). In this study, the cholic acid content of the IO cows was lower than that of the E cows, suggesting that cholic acid intake may have affected the level of cholesterol in the IO cows, which would subsequently affect the synthesis of steroid hormones and thus affect normal follicular development. Therefore, one of the reasons for the occurrence of IO in dairy cows may be that the fall in plasma arachidic acid and cholic acid levels caused by NEB hindered the growth and development of follicles.

Effect of protein metabolism on IO. Leucine, isoleucine, and valine, which are collectively referred to as branched chain amino acids (BCAAs), stimulate the production of insulin, growth hormone, and IGF-1. The average daily milk yield was higher, BCS was lower, and NEFA levels were higher in the IO cows, indicating that the mobilisation of fats and proteins reduced the $\mathrm{BCS}$ due to the energy requirement for lactation (15). Protein synthesis and decomposition are in a state of dynamic equilibrium in the body. In the IO cows, the content of $\gamma$-L-glutamyl-L-valine, an intermediate product of protein degradation, was higher, suggesting that the protein degradation rate was greater than the synthesis rate. Insulin can inhibit the decomposition of fats and proteins and promote their synthesis, but the mobilisation of fats and proteins in IO cows was increased, suggesting low insulin levels or a state of insulin resistance. Insulin and IGF-1 are synthesised from BCAAs. In NEB cows, the BCAA content was increased due to protein decomposition, which inhibited the synthesis of insulin and IGF-1 (14). IGF-1 can synergise with FSH and LH to promote the expression of FSH and LH receptors, thereby promoting follicular development. IGF-1 can also promote the transformation of androgen to $E_{2}$, while promoting the proliferation of granulosa cells and subsequent follicular development (16). NEB cows had lower insulin and Glu levels, and both in vivo and in vitro studies of bovine follicular cells have shown that insulin promotes follicular development $(22,24)$.

Effect of glycometabolism on IO. Mammalian follicles utilise Glu mainly through the glycolysis pathway (26). The phosphofructokinase activity of oocytes is inhibited in a state of weakened glycolysis. Cumulus cells have relatively robust glycolytic activities, in which Glu can be metabolised into pyruvate. Pyruvate provides energy for the development of oocytes by generating acetyl-CoA via the tricarboxylic acid cycle (7). In the cumulus oocyte complex, some Glu enters the pentose phosphate pathway to produce nicotinamide adenine dinucleotide phosphate, which is a hydrogen donor in the synthesis of various substances, such as fatty acids, sex hormones, and nucleic acids. (10). In this study, the contents of L-alanine, L-glutamic acid, D-proline, L-isoleucine, and L-valine, which are all glycogenic amino acids, were increased in the IO cows, suggesting that a large amount of amino acids may be produced by protein mobilisation or amino acid accumulation, resulting from weakened or obstructed amino acid glycogenesis caused by NEB.

Effect of GSH metabolism on IO. L-pyroglutamic acid and L-glutamate are involved in GSH metabolism 
via the $\gamma$-glutamyl cycle, and the intracellular content level of this hormone is an important indicator of oocyte maturation in cell culture. Park et al. (19) found that the level of GSH in bovine follicles was higher in large follicles and Basini et al. (3) reported that the content of reactive oxygen species (ROS) was the highest in small swine follicles and that with the growth of follicles, ROS gradually decreased and antioxidant activity gradually increased. GSH is an important antioxidant that protects cells from oxidative stress during growth. In this study, L-pyroglutamate and L-glutamate were closely related to GSH synthesis. L-pyroglutamate is a downstream metabolite of GSH metabolism and L-glutamate is an important amino acid for the synthesis of GSH. Both were increased in the IO cows, suggesting that NEB caused the proteolysis and metabolic decomposition of GSH to be accelerated, resulting in deficient GSH synthesis, which may have caused the IO in the dairy cows.

Relationship of validated DMs with energy metabolites. The level of cholic acid can to a certain extent reflect the in vivo level of cholesterol, which is the precursor molecule of all steroid hormones, including $\mathrm{E}_{2}$ and $\mathrm{P}_{4}$. Alanine, a nonessential amino acid, is an important energy source that is mainly derived from BCAAs produced by the catabolism of skeletal muscle tissue (29). L-alanine was significantly correlated with NEFA, which is an important indicator of fat mobilisation (1). In energy deficiency or energy metabolism disorders, fat is mobilised to meet energy needs. The increase in NEFA indicated increased fat mobilisation due to energy metabolism disorders, so there exists a certain correlation between L-alanine and NEFA (8). L-pyroglutamic acid is involved in GSH metabolism, and is a downstream product of GSH transport amino acids. This acid was slightly negatively correlated with BHBA, which might have been caused by oxidative stress induced by an increase in BHBA. Glutamate is an important amino acid and plays a key role in amino acid metabolism by providing amino groups for the formation of other amino acids via transamination or deamination of 2-ketoglutaric acid, which is also involved in GSH metabolism. D-proline is involved in the metabolism of arginine and proline, as well as synthesis of arginine, a key substance involved in the urea cycle. The metabolic abnormalities of D-proline indirectly illustrate dysfunction of the urea cycle (18). In addition, L-alanine, L-glutamate, and D-proline are glycogenic amino acids, which were all increased in the IO cows, reflecting that NEB caused the energy metabolism disorders. Also, amino acids are produced by proteolysis, thus an increase in the above amino acids in IO cows explains the enhanced proteolysis in cows to a certain extent (13).

In conclusion, in this study 20 differential plasma metabolites of IO cows were screened by LC-MS, findings including a decrease in cholic acid, p-chlorophenylalanine, and arachidonic acid, and an increase in tyramine, betaine, L-phenylalanine,
L-glutamate, D-proline, L-alanine, and L-pyrophosphate compared to E cows. Five DMs (cholic acid, D-proline, L-glutamate, L-alanine, and L-pyroglutamic acid) were validated by ELISA and found to be in accordance with the results of LC-MS analysis. The screened DMs may be beneficial by facilitating more accurate diagnosis of IO in dairy cows. The metabolic pathways of DMs related to IO were identified and networks were established. However, the specific relationships between DMs and IO require further studies at the molecular level, which will provide a new basis for further exploration of the pathogenesis and prevention of IO in cows in the future.

Conflict of Interests Statement: The authors declare that there is no conflict of interests regarding the publication of this article.

Financial Disclosure Statement: This work was supported by the National Key R\&D Program of China (2017YFD0502200), the National Science Foundation Committee of China (31772804), and Heilongjiang Provincial Key Laboratory of Prevention and Control of Bovine Diseases (PCBD201703).

Animal Rights Statement: The study protocol was approved by the Ethics Committee on the Use and Care of Animals of Heilongjiang Bayi Agricultural University (Daqing, China). All experimental animals were treated according to the International Guiding Principles for Biomedical Research Involving Animals (Pan American Health Organisation, 2010).

Acknowledgments: The authors would like to thank International Science Editing.

* Yu-Xi Song and Pan Hu made equal contributions to this work.

\section{References}

1. Adewuyi A.A., Gruys E., van Eerdenburg F.J.: Non esterified fatty acids (NEFA) in dairy cattle. Vet Q 2005, 27, 117-126.

2. Ahmed W.M.: Overview on some factors negatively affecting ovarian activity in large farm animals. Global Vet 2007, 1, 53-66.

3. Basini G., Simona B., Santini S.E., Grasselli F.: Reactive oxygen species and anti-oxidant defences in swine follicular fluids. Reprod Fert Develop 2008, 20, 269-274.

4. Benton H.P., Ivanisevic J., Mahieu N., Kurczy M.E., Johnson C.H., Franco L., Rinehart D., Valentine E., Gowda H., Ubhi B.: Autonomous metabolomics for rapid metabolite identification in global profiling. Anal Chem 2015, 87, 884-891.

5. Bremer J.: Carnitine - metabolism and functions. Physiol Rev 1983, 63, 1420-1480.

6. Butler S.T., Pelton S.H., Butler W.R.: Energy balance, metabolic status, and the first postpartum ovarian follicle wave in cows administered propylene glycol. J Dairy Sci 2006, 89, 2938-2951.

7. Cetica P., Pintos L., Dalvit G., Beconi M.: Activity of key enzymes involved in glucose and triglyceride catabolism during bovine oocyte maturation in vitro. Reproduction 2002, 124, 675-681. 
8. Forde N., O'Gorman A., Whelan H., Duffy P., O'Hara L., Kelly A.K., Lonergan P.: Lactation-induced changes in metabolic status and follicular-fluid metabolomic profile in postpartum dairy cows. Reprod Fert Develop 2016, 28, 1882-1892.

9. Fortune J.E.: The early stages of follicular development: activation of primordial follicles and growth of preantral follicles. Anim Reprod Sci 2003, 78, 135-163.

10. Gutnisky C., Dalvit G.C., Thompson J.G., Cetica P.D.: Pentose phosphate pathway activity: effect on in vitro maturation and oxidative status of bovine oocytes. Reprod Fert Develop 2014, 26, 931-942.

11. Hassan A.S., Gallon L.S., Zimmer L.A., Balistreri W.F., Subbiah M.T.R.: Persistent enhancement of bile acid synthesis in guinea pigs following stimulation of cholesterol catabolism in neonatal life. Steroids 1981, 38, 477-484.

12. Ivanisevic J., Elias D., Deguchi H., Averell P.M., Kurczy M., Johnson C.H., Tautenhahn R., Zhu Z., Watrous J., Jain M., Griffin J., Patti G.J., Siuzdak G.: Arteriovenous blood metabolomics: a readout of intra-tissue metabostasis. Sci Rep-UK 2015, 5, 12757.

13. Li M., Ruan L.Y., Liu Y., Xu H.D., Chen T., Fu Y.H., Jiang L., Wang J.S.: Insight into biological system responses in goldfish (Carassius auratus) to multiple doses of avermectin exposure by integrated 1H NMR-based metabolomics. Toxicol Res-UK 2015, 4, 1374-1388.

14. Lucy M.C.: Functional differences in the growth hormone and insulin-like growth factor axis in cattle and pigs: implications for post-partum nutrition and reproduction. Reprod Domest Anim 2008, 43, 31-39.

15. Maillo V., Rizos D., Besenfelder U., Havlicek V., Kelly A.K., Garrett M., Lonergan P.: Influence of lactation on metabolic characteristics and embryo development in postpartum Holstein dairy cows. J Dairy Sci 2012, 95, 3865-3876.

16. Martinez-Chequer J.C., Stouffer R.L., Hazzard T.M., Patton P.E., Molskness T.A.: Insulin-like growth factors-1 and -2 , but not hypoxia, synergize with gonadotropin hormone to promote vascular endothelial growth factor-A secretion by monkey granulosa cells from preovulatory follicles. Biol Reprod 2003, 68, 1112-1118.

17. McLaughlin E.A., McIver S.C.: Awakening the oocyte: controlling primordial follicle development. Reproduction 2009, $137,1-11$.

18. Morris S.M.: Regulation of enzymes of the urea cycle and arginine metabolism. Annu Rev Nutr 2002, 22, 87-105.

19. Park S.H., Cho H.S., Yu I.J.: Effect of bovine follicular fluid on reactive oxygen species and glutathione in oocytes, apoptosis and apoptosis-related gene expression of in vitro-produced blastocysts. Reprod Domest Anim 2014, 49, 370-377.

20. Peter A.T., Vos P.L.A.M., Ambrose D.J.: Postpartum anestrus in dairy cattle. Theriogenology 2009, 71, 1333-1342.

21. Salesh N., Mahmud E., Waded E.: Interactions between insulin like growth factor 1, thyroid hormones and blood energy metabolites in cattle with postpartum inactive ovaries. Nat Sci 2011, 9, 56-63.

22. Simpson R.B., Chase C.C Jr., Spicer L.J., Vernon R.K., Hammond A.C., Rae D.O.: Effect of exogenous insulin on plasma and follicular insulin-like growth factor I, insulin-like growth factor binding protein activity, follicular oestradiol and progesterone, and follicular growth in superovulated Angus and Brahman cows. J Reprod Ferti 1994, 102, 483-492.

23. Smith C.A., Want E.J., O’Maille G., Abagyan R., Siuzdak G.: XCMS: processing mass spectrometry data for metabolite profiling using nonlinear peak alignment, matching, and identification. Anal Chem 2006, 78, 779-787.

24. Spicer L.J., Alpizar E., Echternkamp S.E.: Effects of insulin, insulin-like growth factor I, and gonadotropins on bovine granulosa cell proliferation, progesterone production, estradiol production, and (or) insulin-like growth factor I production in vitro. J Anim Sci 1993, 71, 1232-1241.

25. Staples C.R., Burke J.M., Thatcher W.W.: Influence of supplemental fats on reproductive tissues and performance of lactating cows. J Dairy Sci 1998, 81, 856-871.

26. Sutton-McDowall M.L., Gilchrist R.B., Thompson J.G.: The pivotal role of glucose metabolism in determining oocyte developmental competence. Reproduction 2010, 139, 685-695.

27. Trygg J., Wold S.: Orthogonal projections to latent structures (O-PLS). J Chemometri 2002, 16, 119-128.

28. Xu C., Xia C., Sun Y., Xiao X., Wang G., Fan Z., Shu S., Zhang H., Xu C., Yang W.: Metabolic profiles using (1)H-nuclear magnetic resonance spectroscopy in postpartum dairy cows with ovarian inactivity. Theriogenology 2016, 86, 1475-1481.

29. Yudkoff M., Daikhin Y., Nissim I., Horyn O., Luhovyy B., Lazarow A., Nissim I.: Brain amino acid requirements and toxicity: the example of leucine. J Nutr 2005, 135, 1531-1538.

30. Zeron Y., Ocheretny A., Kedar O., Borochov A., Sklan D., Arav A.: Seasonal changes in bovine fertility: relation to developmental competence of oocytes, membrane properties, and fatty acid composition of follicles. Reproduction 2001, 121, 447-454. 